\title{
Diffractive Dijet Production at HERA
}

\author{
A. Bruni ${ }^{1}$, M. Klasen ${ }^{2,3}$, G. Kramer ${ }^{3}$ and S. Schätzel ${ }^{4}$ \\ ${ }^{1}$ INFN Bologna, Via Irnerio 46, 40156 Bologna, Italy \\ ${ }^{2}$ Laboratoire de Physique Subatomique et de Cosmologie, Université Joseph Fourier/CNRS-IN2P3, 53 \\ Avenue des Martyrs, 38026 Grenoble, France \\ ${ }^{3}$ II. Inst. für Theoret. Physik, Universität Hamburg, Luruper Chaussee 149, 22761 Hamburg, Germany \\ ${ }^{4}$ DESY FLC, Notkestr. 85, 22607 Hamburg, Germany
}

\begin{abstract}
We present recent experimental data from the H1 and ZEUS Collaborations at HERA for diffractive dijet production in deep-inelastic scattering (DIS) and photoproduction and compare them with next-to-leading order (NLO) QCD predictions using diffractive parton densities. While good agreement is found for DIS, the dijet photoproduction data are overestimated by the NLO theory, showing that factorization breaking occurs at this order. While this is expected theoretically for resolved photoproduction, the fact that the data are better described by a global suppression of direct and resolved contribution by about a factor of two comes as a surprise. We therefore discuss in some detail the factorization scheme and scale dependence between direct and resolved contributions and propose a new factorization scheme for diffractive dijet photoproduction.
\end{abstract}

\section{Introduction}

It is well known that in high-energy deep-inelastic ep-collisions a large fraction of the observed events are diffractive. These events are defined experimentally by the presence of a forward-going system $Y$ with four-momentum $p_{Y}$, low mass $M_{Y}$ (in most cases a single proton and/or low-lying nucleon resonances), small momentum transfer squared $t=\left(p-p_{Y}\right)^{2}$, and small longitudinal momentum transfer fraction $x_{\mathbb{P}}=q\left(p-p_{Y}\right) / q p$ from the incoming proton with four-momentum $p$ to the system $X$ (see Fig. 1).

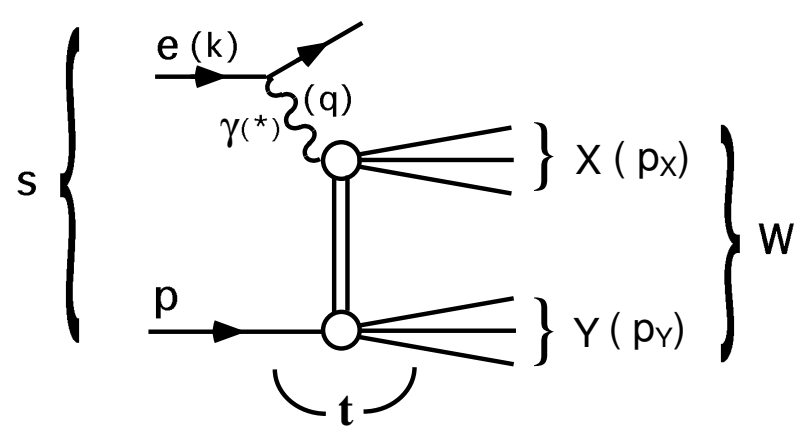

Fig. 1: Diffractive scattering process $e p \rightarrow e X Y$, where the hadronic systems $X$ and $Y$ are separated by the largest rapidity gap in the final state.

The presence of a hard scale, as for example the photon virtuality $Q^{2}=-q^{2}$ in deep-inelastic scattering (DIS) or the large transverse jet momentum $p_{T}^{*}$ in the photon-proton centre-of-momentum frame, should then allow for calculations of the production cross section for the central system $X$ with the known methods of perturbative QCD. Under this assumption, the cross section for the inclusive production of two jets, $e+p \rightarrow e+2$ jets $+X^{\prime}+Y$, can be predicted from the well-known formulæ for jet production 
in non-diffractive $e p$ collisions, where in the convolution of the partonic cross section with the parton distribution functions (PDFs) of the proton the latter ones are replaced by the diffractive PDFs. In the simplest approximation, they are described by the exchange of a single, factorizable pomeron/Reggepole.

The diffractive PDFs have been determined by the H1 Collaboration at HERA from high-precision inclusive measurements of the DIS process $e p \rightarrow e X Y$ using the usual DGLAP evolution equations in leading order (LO) and next-to-leading order (NLO) and the well-known formula for the inclusive cross section as a convolution of the inclusive parton-level cross section with the diffractive PDFs [1]. For a similar analysis of the inclusive measurements of the ZEUS Collaboration see [2,3]. A longer discussion of the extraction of diffractive PDFs can also be found in these proceedings [4] and in [5]. For inclusive diffractive DIS it has been proven by Collins that the formula referred to above is applicable without additional corrections and that the inclusive jet production cross section for large $Q^{2}$ can be calculated in terms of the same diffractive PDFs [6]. The proof of this factorization formula, usually referred to as the validity of QCD factorization in hard diffraction, may be expected to hold for the direct part of photoproduction $\left(Q^{2} \simeq 0\right)$ or low- $Q^{2}$ electroproduction of jets [6]. However, factorization does not hold for hard processes in diffractive hadron-hadron scattering. The problem is that soft interactions between the ingoing two hadrons and their remnants occur in both the initial and final state. This agrees with experimental measurements at the Tevatron [7]. Predictions of diffractive dijet cross sections for $p \bar{p}$ collisions as measured by CDF using the same PDFs as determined by $\mathrm{H} 1$ [1] overestimate the measured cross section by up to an order of magnitude [7]. This suppression of the CDF cross section can be explained by considering the rescattering of the two incoming hadron beams which, by creating additional hadrons, destroy the rapidity gap [8].

Processes with real photons $\left(Q^{2} \simeq 0\right)$ or virtual photons with fixed, but low $Q^{2}$ involve direct interactions of the photon with quarks from the proton as well as resolved photon contributions, leading to parton-parton interactions and an additional remnant jet coming from the photon (for a review see [9]). As already said, factorization should be valid for direct interactions as in the case of DIS, whereas it is expected to fail for the resolved process similar as in the hadron-hadron scattering process. In a two-channel eikonal model similar to the one used to calculate the suppression factor in hadron-hadron processes [8], introducing vector-meson dominated photon fluctuations, a suppression by about a factor of three for resolved photoproduction at HERA is predicted [10]. Such a suppression factor has recently been applied to diffractive dijet photoproduction [11,12] and compared to preliminary data from H1 [13] and ZEUS [14]. While at LO no suppression of the resolved contribution seemed to be necessary, the NLO corrections increase the cross section significantly, showing that factorization breaking occurs at this order at least for resolved photoproduction and that a suppression factor $R$ must be applied to give a reasonable description of the experimental data.

As already mentioned elsewhere $[11,12]$, describing the factorization breaking in hard photoproduction as well as in electroproduction at very low $Q^{2}$ [15] by suppressing the resolved contribution only may be problematic. An indication for this is the fact that the separation between the direct and the resolved process is uniquely defined only in LO. In NLO these two processes are related. The separation depends on the factorization scheme and the factorization scale $M_{\gamma}$. The sum of both cross sections is the only physically relevant cross section, which is approximately independent of the factorization scheme and scale [16]. As demonstrated in Refs. [11,12] multiplying the resolved cross section with the suppression factor $R=0.34$ destroys the correlation of the $M_{\gamma}$-dependence between the direct and resolved part, and the sum of both parts has a stronger $M_{\gamma}$-dependence than for the unsuppressed case ( $R=1$ ), where the $M_{\gamma}$-dependence of the NLO direct cross section is compensated to a high degree against the $M_{\gamma}$-dependence of the LO resolved part.

In the second Section of this contribution, we present the current experimental data from the $\mathrm{H} 1$ and ZEUS Collaborations on diffractive dijet production in DIS and photoproduction and compare these data to theoretical predictions at NLO for two different scenarios: suppression of only the resolved 
part by a factor $R=0.34$ as expected from LO theory and proposed in [8], and equal suppression of all direct and resolved contributions by a factor $R=0.5$, which appears to describe the data better phenomenologically. This motivates us to investigate in the third Section the question whether certain parts of the direct contribution might break factorization as well and therefore need a suppression factor.

The introduction of the resolved cross section is dictated by perturbation theory. At NLO, collinear singularities arise from the photon initial state, which are absorbed at the factorization scale into the photon PDFs. This way the photon PDFs become $M_{\gamma}$-dependent. The equivalent $M_{\gamma}$-dependence, just with the opposite sign, is left in the NLO corrections to the direct contribution. With this knowledge, it is obvious that we can obtain a physical cross section at NLO, i.e. the superposition of the NLO direct and LO resolved cross section, with a suppression factor $R<1$ and no $M_{\gamma}$-dependence left, if we also multiply the $\ln M_{\gamma}$-dependent term of the NLO correction to the direct contribution with the same suppression factor as the resolved cross section. We are thus led to the theoretical conclusion that, contrary to what one may expect, not all parts of the direct contribution factorize. Instead, the initial state singular part appearing beyond LO breaks factorization even in direct photoproduction, presumably through soft gluon attachments between the proton and the collinear quark-antiquark pair emerging from the photon splitting. This would be in agreement with the general remarks about initial state singularities in Ref. [6].

In the third Section of this contribution, we present the special form of the $\ln M_{\gamma}$-term in the NLO direct contribution and demonstrate that the $M_{\gamma}$-dependence of the physical cross section cancels to a large extent in the same way as in the unsuppressed case $(R=1)$. These studies can be done for photoproduction $\left(Q^{2} \simeq 0\right)$ as well as for electroproduction with fixed, small $Q^{2}$. Since in electroproduction the initial-state singularity in the limit $Q^{2} \rightarrow 0$ is more directly apparent than for the photoproduction case, we shall consider in this contribution the low- $Q^{2}$ electroproduction case just for demonstration. This diffractive dijet cross section has been calculated recently [15]. A consistent factorization scheme for low- $Q^{2}$ virtual photoproduction has been defined and the full (direct and resolved) NLO corrections for inclusive dijet production have been calculated in [17]. In this work we adapt this inclusive NLO calculational framework to diffractive dijet production at low- $Q^{2}$ in the same way as in [15], except that we multiply the $\ln M_{\gamma}$-dependent terms as well as the resolved contributions with the same suppression factor $R=0.34$, as an example, as in our earlier work $[11,12,15]$. The exact value of this suppression factor may change in the future, when better data for photoproduction and low- $Q^{2}$ electroproduction have been analyzed. We present the $\ln M_{\gamma}$-dependence of the partly suppressed NLO direct and the fully suppressed NLO resolved cross section $\mathrm{d} \sigma / \mathrm{d} Q^{2}$ and their sum for the lowest $Q^{2}$ bin, before we give a short summary in section 4 .

\section{Comparison of $\mathrm{H1}$ and ZEUS Data with NLO Theory Predictions}

In this Section, diffractive PDFs [1-3] extracted from diffractive structure function data are used in NLO calculations to test factorisation in diffractive dijet production. Dijet production is directly sensitive to the diffractive gluon (Fig. 2) whereas in inclusive measurements the gluon is determined from scaling violations.

\subsection{Diffractive Dijet Production in DIS}

$\mathrm{H} 1$ has measured the cross sections for dijet production [13] in the kinematic range $Q^{2}>4 \mathrm{GeV}^{2}$, $165<W<242 \mathrm{GeV}$ (photon-proton centre-of-mass energy) and $x_{\mathbb{P}}<0.03$. Jets are identified using the inclusive $k_{T}$ cluster algorithm and selected by requiring $E_{T}^{*, j e t}(1,2)>5,4 \mathrm{GeV}$ and $-3<\eta_{\text {jet }}^{*}<0 .{ }^{1}$ NLO predictions have been obtained by interfacing the H1 diffractive PDFs with the DISENT program [18]. The renormalisation and factorisation scales were set to the transverse energy of the leading parton jet. The NLO parton jet cross sections have been corrected for hadronisation effects using the

\footnotetext{
${ }^{1}$ The ' $*$ ' denotes variables in the photon-proton centre-of-mass system.
} 

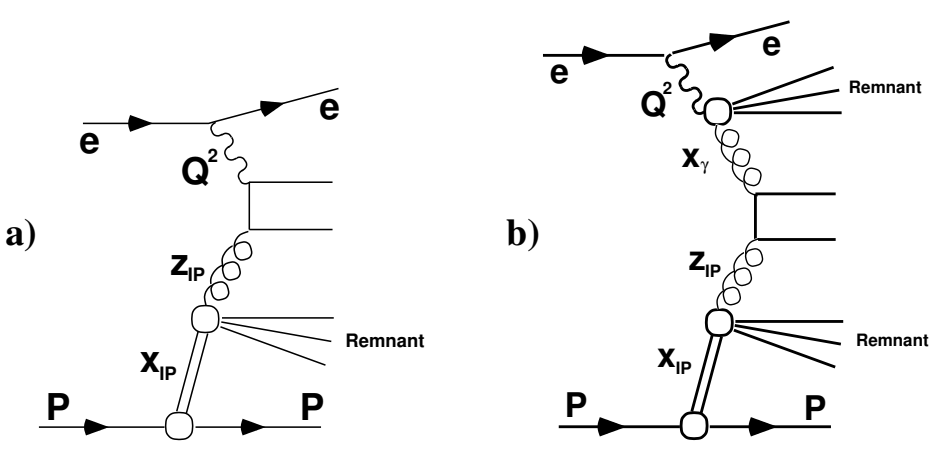

Fig. 2: Example processes for a) direct photon and b) resolved photon interactions.

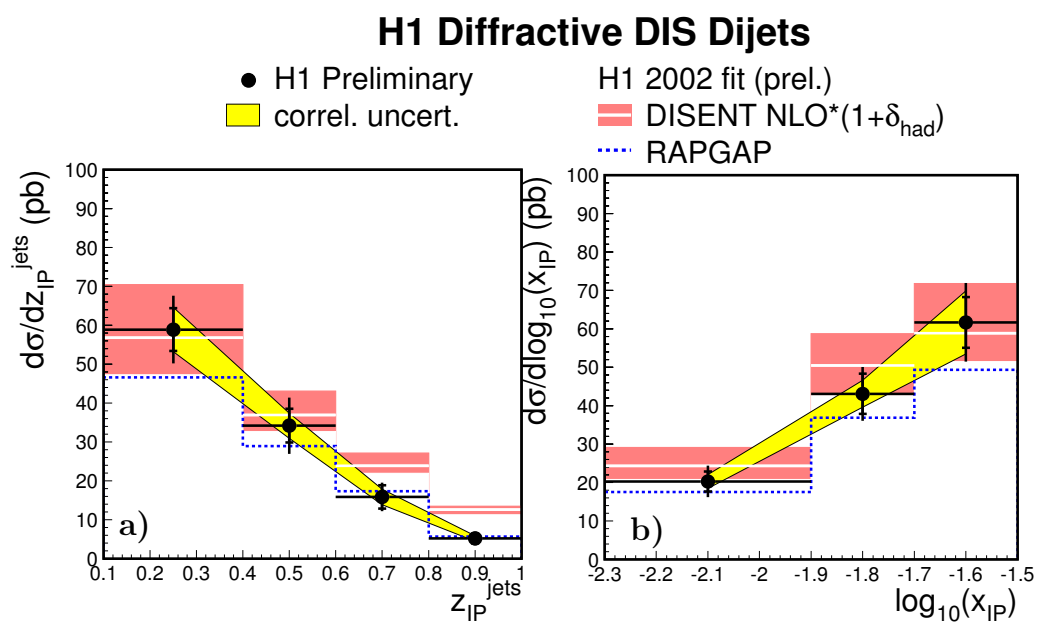

Fig. 3: Diffractive DIS dijet cross sections compared with a NLO prediction based on diffractive PDFs and with RAPGAP.

leading order (LO) generator RAPGAP [19] with parton showers and the Lund string fragmentation model. Comparisons of the DISENT and RAPGAP predictions with the measured cross section differential in $z_{\mathbb{P}}^{\text {jets }}$, an estimator for the fraction of the momentum of the diffractive exchange entering the hard scatter, are shown in Fig. 3a. The inner band around the NLO calculation indicates the $\approx 20 \%$ uncertainty resulting from a variation of the renormalisation scale by factors 0.5 and 2 . The uncertainty in the diffractive PDFs is not shown. Within this additional uncertainty, which is large at high $z_{\mathbb{P}}^{\text {jets }}$, the cross section is well described. The cross section differential in $\log _{10}\left(x_{\mathbb{P}}\right), p_{T}^{\text {jet1 }}$, and $Q^{2}$ is shown in Figs. 3b and 4. All distributions are well described and QCD factorisation is therefore in good agreement with dijet production in diffractive DIS.

Similar results are presented by ZEUS [20]; the dijet cross sections have been measured in the kinematic range $5<Q^{2}<100 \mathrm{GeV}^{2}, 100<W<200 \mathrm{GeV}, x_{\mathbb{P}}<0.03$. The jets were identified using the inclusive $k_{T}$ algorithm in the $\gamma p$ frame and required to satisfy $E_{T}^{* \text {,jet }}(1,2)>5,4 \mathrm{GeV}$ and $-3.5<\eta_{\mathrm{jet}}^{*}<0.0$. NLO predictions have been obtained with the DISENT program interfaced to three different sets of diffractive PDFs: from fit to H1 data [1], from fit to the ZEUS $M_{X}$ data (GLP) [3] and from fit to ZEUS LPS and $F_{2}^{D \text {,charm }}$ data [2]. Comparisons of the DISENT predictions with the measured cross section differential in $E_{T}^{* \text {,jet }}, \eta_{\text {jet }}^{*}, z_{\mathbb{P}}^{\text {jets }}$ and $x_{\gamma}^{\text {obs }}$ are shown in Fig. 5. The $20-30 \%$ uncertainty in the NLO calculations resulting from a variation of the renormalisation and factorisation scales is not shown. Within the experimental and QCD scale uncertainties, the predictions based on the H1 and ZEUS-LPS PDFs give a good description of the dijet cross section. The normalisation of the prediction using the GLP fit is substantially lower than those from the other two sets of PDFs. For 


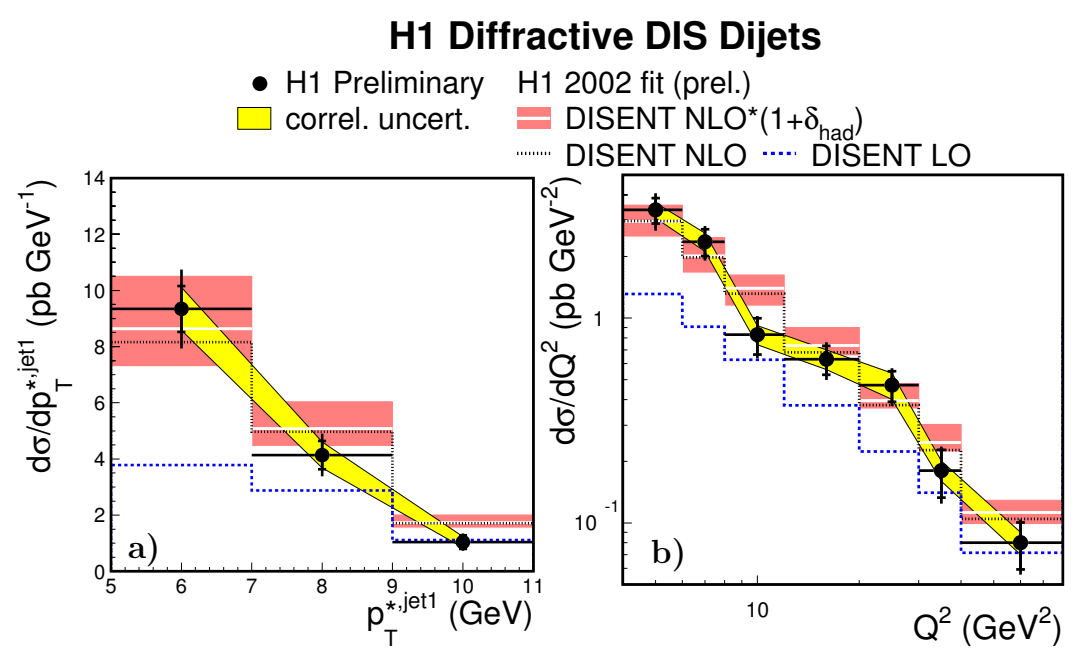

Fig. 4: Diffractive DIS dijet cross sections compared with a NLO prediction based on diffractive PDFs.
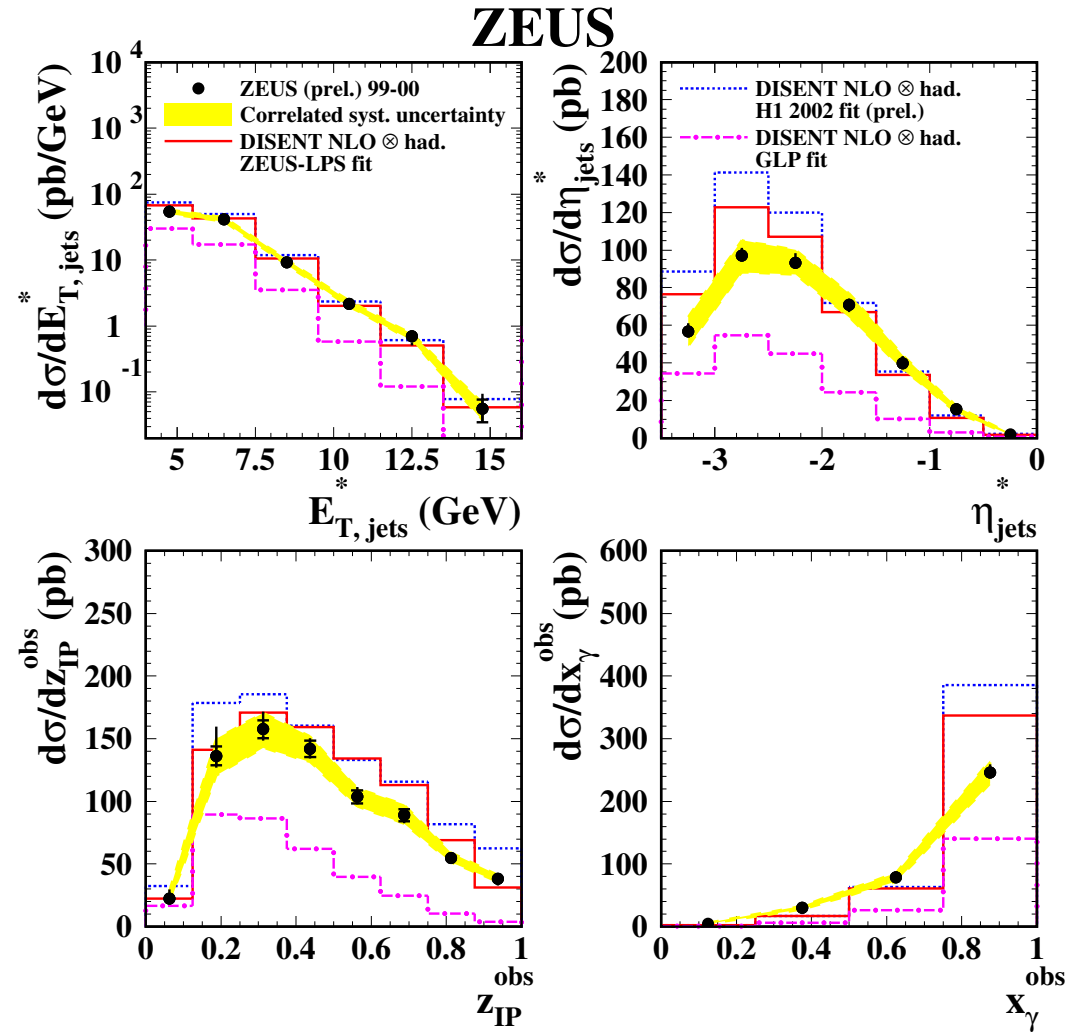

Fig. 5: Diffractive DIS dijet cross sections compared with NLO predictions based on three sets of diffractive PDFs.

ZEUS, the difference observed between the three sets may be interpreted as an estimate of the uncertainty associated with the diffractive PDFs and with the definition of the diffractive region. The dijet data could be included in future fits in order to better constrain the diffractive gluon distribution.

Within the experimental and theoretical uncertainties and assuming the H1 diffractive PDFs, factorisation is in good agreement with diffractive $D^{*}$ production $[21,22]$ in the DIS kinematic region. 


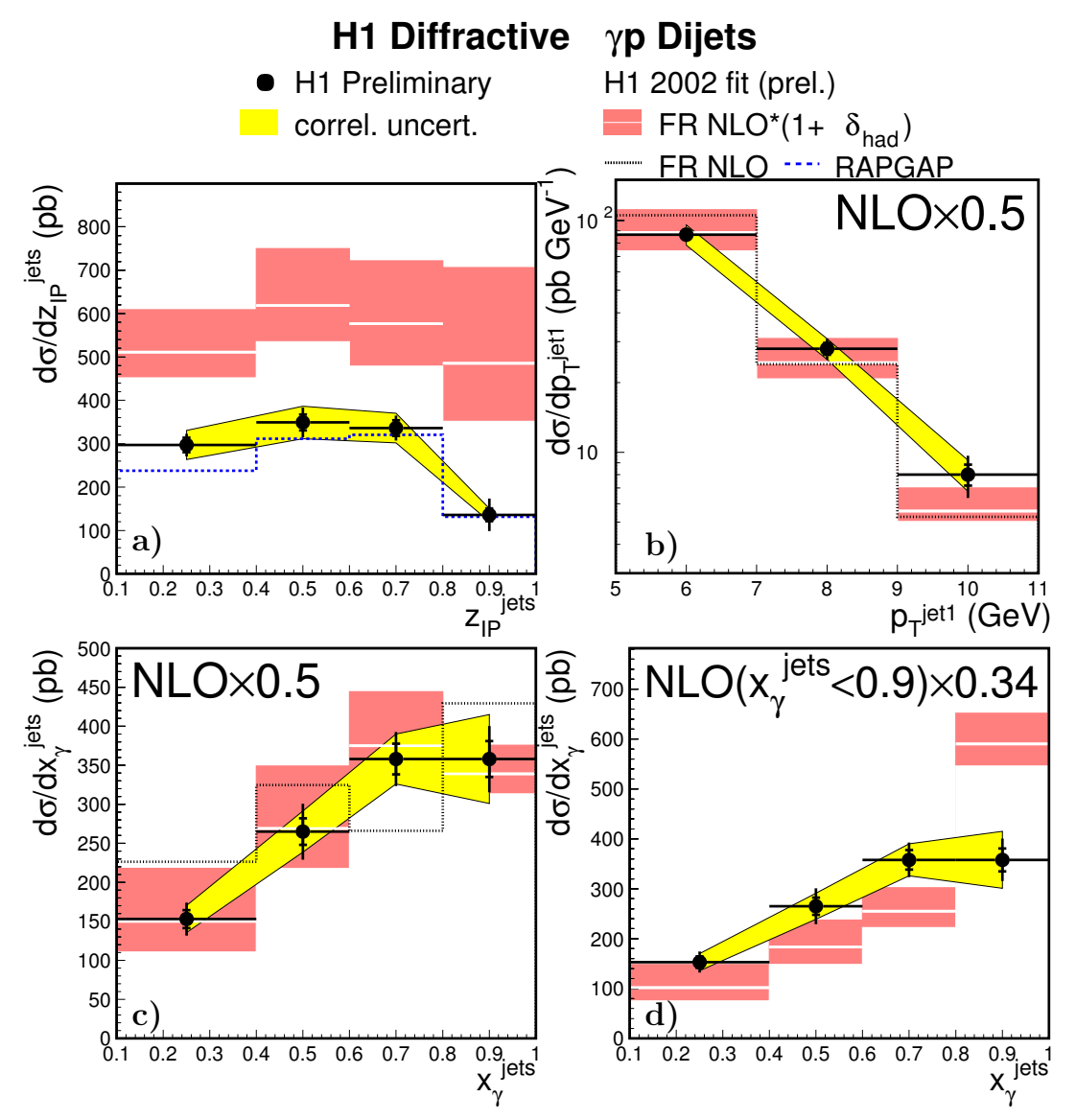

Fig. 6: a) Diffractive dijet photoproduction cross section differential in $z_{\mathbb{P}}^{\text {jets }}$ compared with a NLO prediction based on diffractive PDFs and RAPGAP. b)-d): Cross section differential in $p_{T}^{\text {jet1 }}$ and $x_{\gamma}^{\text {jets }}$, compared with the NLO prediction modified as follows: in b) and c) the calculation is scaled by a global factor 0.5 whereas in d) only the "resolved" part is scaled by 0.34 .

\subsection{Diffractive Photoproduction of Dijets}

In photoproduction, a sizeable contribution to the cross section is given by resolved photon processes (Fig. 2b) in which only a fraction $x_{\gamma}<1$ of the photon momentum enters the hard scatter. The photoproduction dijet cross section measured by $\mathrm{H} 1\left(Q^{2}<0.01 \mathrm{GeV}^{2}, 165<W<242 \mathrm{GeV}, x_{\mathbb{P}}<0.03\right.$, $E_{T}^{\text {jet }}(1,2)>5,4 \mathrm{GeV},-1<\eta_{\text {jet }}<2$, inclusive $k_{T}$ algorithm) is shown in Fig. 6 [13]. NLO predictions have been obtained with the Frixione et al. program [23] interfaced to the H1 diffractive PDFs. The parton jet calculation is corrected for hadronisation effects using RAPGAP. The cross section differential in $z_{\mathbb{P}}^{\text {jets }}$ is shown in Fig. $6 \mathrm{a}$. The calculation lies a factor $\approx 2$ above the data. Fig. $6 \mathrm{~b}$ and $6 \mathrm{c}$ show the cross section as a function of $p_{T}^{\text {jet1 }}$ and $x_{\gamma}^{\text {jets }}$ and the NLO predictions have been scaled down by a factor 0.5. Good agreement is obtained for this global suppression. In Fig. 6d, only the "resolved" part for which $x_{\gamma}^{\text {jets }}<0.9$ at the parton level is scaled by a factor 0.34 . This factor was proposed by Kaidalov et al. [10] for the suppression of the resolved part in LO calculations. The calculation for $x_{\gamma}^{\text {jets }}>0.9$ is left unscaled. This approach is clearly disfavoured.

The ZEUS measurement [24] $\left(Q^{2}<0.01 \mathrm{GeV}^{2}, x_{\mathbb{P}}<0.025,0.2<y<0.85, E_{T}^{\mathrm{jet}}(1,2)>\right.$ $7.5,6.5 \mathrm{GeV},-1.5<\eta<1.5$, inclusive $k_{T}$ algorithm) is shown in Figs. 7 and 8 separately for samples enriched in "direct" $\left(x_{\gamma}^{\text {jets }}>0.75\right)$ and "resolved" $\left(x_{\gamma}^{\text {jets }}<0.75\right)$ processes, respectively. The NLO [12] prediction using the $\mathrm{H} 1$ diffractive PDFs is also presented corrected for hadronization effects and with the "resolved" part scaled by the factor 0.34. No evidence is observed for a suppression of resolved photon processes relative to direct photon processes in any particular kinematic region. 


\section{ZEUS}
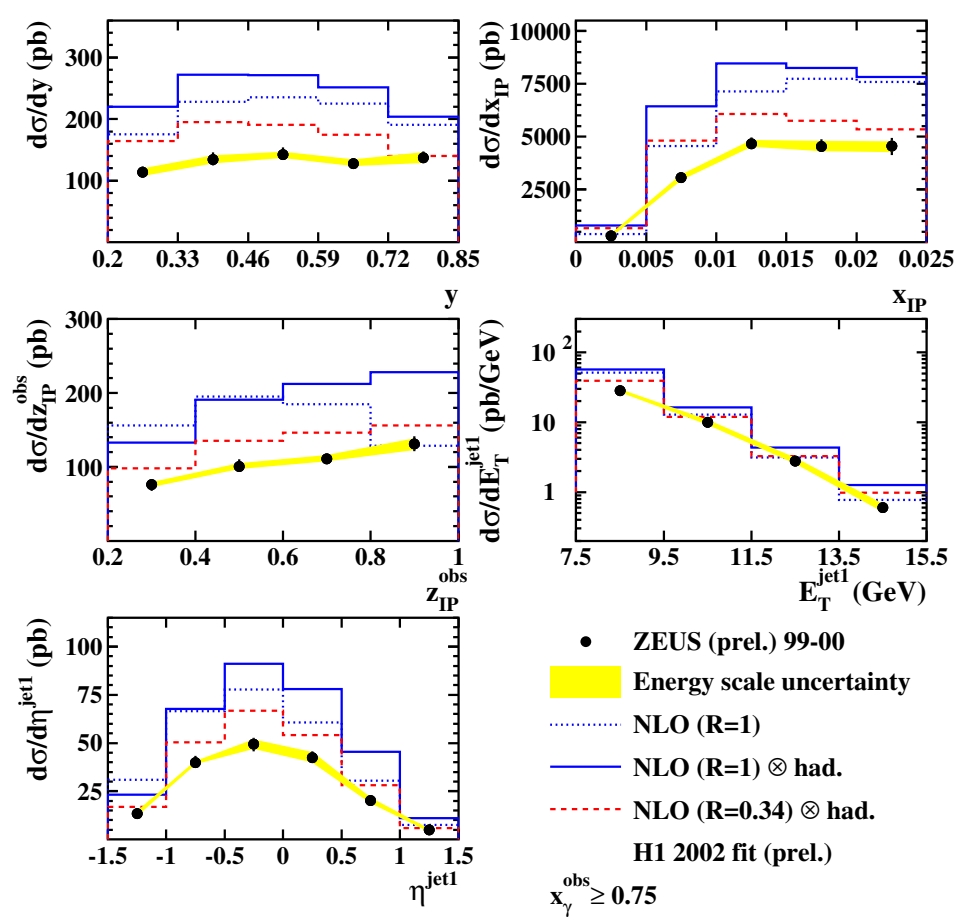

- ZEUS (prel.) 99-00

Energy scale uncertainty

….......... NLO $(\mathbf{R}=\mathbf{1})$

NLO $(R=1) \otimes$ had.

- . - - - NLO $(R=0.34) \otimes$ had.

H1 2002 fit (prel.)

$\mathbf{x}_{\gamma}^{\text {obs }} \geq \mathbf{0 . 7 5}$

Fig. 7: Direct enriched photoproduction. Diffractive dijet photoproduction cross section differential in $y, x_{\mathbb{P}}$, $z_{\mathbb{P}}^{\text {jets }}, E_{T}^{\text {jet }}{ }^{1}$ and $\eta_{\text {jet }}{ }_{1}$ compared with a NLO prediction based on diffractive PDFs. The NLO prediction is also presented corrected for hadronization effects and with the "resolved" part scaled by 0.34 .

Diffractive dijet photoproduction is overestimated by calculations based on PDFs which give a good description of the diffractive DIS data. Factorisation is broken in photoproduction relative to DIS by a factor $\approx 0.5$ with no observed dependence on $x_{\gamma}$ or other kinematic variables.

\section{Factorization and its Breaking in Diffractive Dijet Production}

The fact that equal suppression of direct and resolved photoproduction by a factor $R=0.5$ appears to describe the H1 and ZEUS data better phenomenologically motivates us to investigate in some detail the question whether certain parts of the direct contribution might break factorization as well and therefore need a suppression factor. These studies can be done for photoproduction $\left(Q^{2} \simeq 0\right)$ as well as for electroproduction with fixed, small $Q^{2}$. Since in electroproduction the initial-state singularity in the limit $Q^{2} \rightarrow 0$ is more directly apparent than for the photoproduction case, we shall consider in this contribution the low- $Q^{2}$ electroproduction case just for demonstration.

A factorization scheme for virtual photoproduction has been defined and the full NLO corrections for inclusive dijet production have been calculated in [17]. They have been implemented in the NLO Monte Carlo program JETVIP [25] and adapted to diffractive dijet production in [15]. The subtraction term, which is absorbed into the PDFs of the virtual photon $f_{a / \gamma}\left(x_{\gamma}, M_{\gamma}\right)$, can be found in [26]. The main term is proportional to $\ln \left(M_{\gamma}^{2} / Q^{2}\right)$ times the splitting function

$$
P_{q_{i} \leftarrow \gamma}(z)=2 N_{c} Q_{i}^{2} \frac{z^{2}+(1-z)^{2}}{2}
$$

where $z=p_{1} p_{2} / p_{0} q \in[x ; 1]$ and $Q_{i}$ is the fractional charge of the quark $q_{i} . p_{1}$ and $p_{2}$ are the momenta of the two outgoing jets, and $p_{0}$ and $q$ are the momenta of the ingoing parton and virtual photon, respec- 


\section{ZEUS}
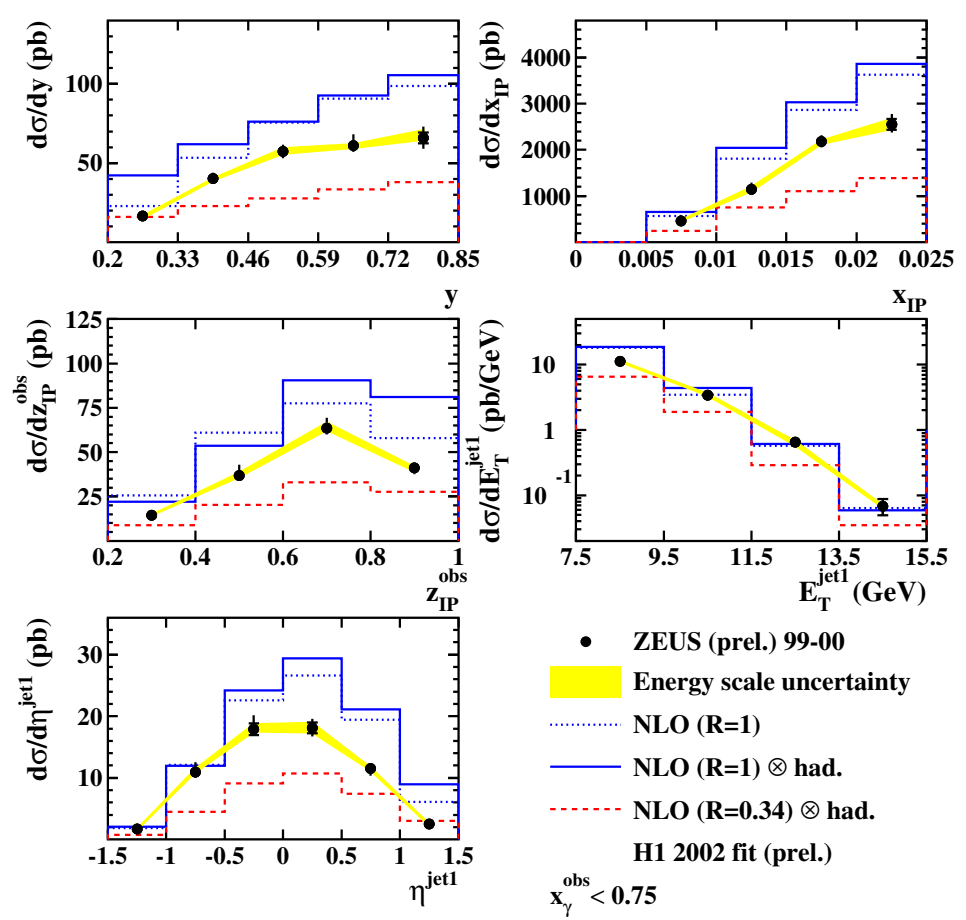

- ZEUS (prel.) 99-00

Energy scale uncertainty

……..... NLO $(\mathbf{R}=\mathbf{1})$

NLO $(R=1) \otimes$ had.

- . - - - NLO $(R=0.34) \otimes$ had.

H1 2002 fit (prel.)

$\mathbf{x}_{\gamma}^{\text {obs }}<\mathbf{0 . 7 5}$

Fig. 8: Resolved enriched photoproduction. Diffractive dijet photoproduction cross section differential in $y, x_{\mathbb{P}}$, $z_{\mathbb{P}}^{\text {jets }}, E_{T}^{\text {jet }^{1}}$ and $\eta_{\text {jet }}$ compared with a NLO prediction based on diffractive PDFs. The NLO prediction is also presented corrected for hadronization effects and with the "resolved" part scaled by 0.34 .

tively. Since $Q^{2}=-q^{2} \ll M_{\gamma}^{2}$, the subtraction term is large and is therefore resummed by the DGLAP evolution equations for the virtual photon PDFs. After this subtraction, the finite term $M\left(Q^{2}\right)_{\overline{\mathrm{MS}}}$, which remains in the matrix element for the NLO correction to the direct process [17], has the same $M_{\gamma^{-}}$ dependence as the subtraction term, i.e. $\ln M_{\gamma}$ is multiplied with the same factor. As already mentioned, this yields the $M_{\gamma}$-dependence before the evolution is turned on. In the usual non-diffractive dijet photoproduction these two $M_{\gamma}$-dependences cancel, when the NLO correction to the direct part is added to the LO resolved cross section [16]. Then it is obvious that the approximate $M_{\gamma}$-independence is destroyed, if the resolved cross section is multiplied by a suppression factor $R$ to account for the factorization breaking in the experimental data. To remedy this deficiency, we propose to multiply the $\ln M_{\gamma}$-dependent term in $M\left(Q^{2}\right)_{\overline{\mathrm{MS}}}$ with the same suppression factor as the resolved cross section. This is done in the following way: we split $M\left(Q^{2}\right)_{\overline{\mathrm{MS}}}$ into two terms using the scale $p_{T}^{*}$ in such a way that the term containing the slicing parameter $y_{s}$, which was used to separate the initial-state singular contribution, remains unsuppressed. In particular, we replace the finite term after the subtraction by

$$
\begin{aligned}
M\left(Q^{2}, R\right)_{\overline{\mathrm{MS}}}= & {\left[-\frac{1}{2 N_{c}} P_{q_{i} \leftarrow \gamma}(z) \ln \left(\frac{M_{\gamma}^{2} z}{p_{T}^{* 2}(1-z)}\right)+\frac{Q_{i}^{2}}{2}\right] R } \\
& -\frac{1}{2 N_{c}} P_{q_{i} \leftarrow \gamma}(z) \ln \left(\frac{p_{T}^{* 2}}{z Q^{2}+y_{s} s}\right),
\end{aligned}
$$

where $R$ is the suppression factor. This expression coincides with the finite term after subtraction (see Ref. [26]) for $R=1$, as it should, and leaves the second term in Eq. (2) unsuppressed. In Eq. (2) we have suppressed in addition to $\ln \left(M_{\gamma}^{2} / p_{T}^{* 2}\right)$ also the $z$-dependent term $\ln (z /(1-z))$, which is specific to the $\overline{\mathrm{MS}}$ subtraction scheme as defined in [17]. The second term in Eq. (2) must be left in its original form, 


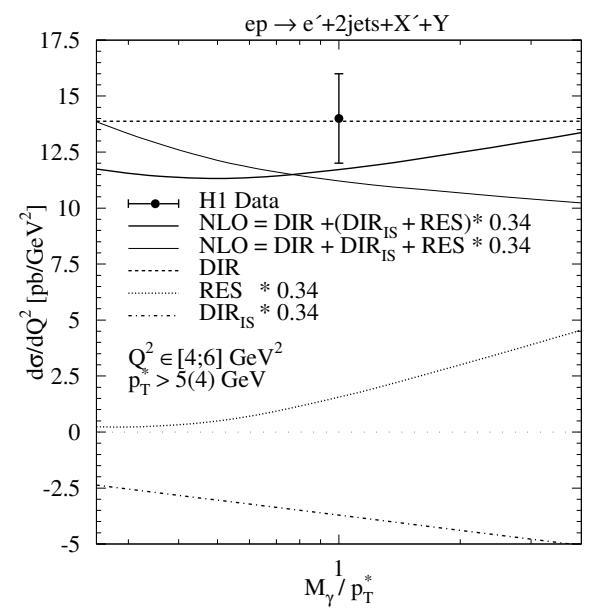

Fig. 9: Photon factorization scale dependence of resolved and direct contributions to $\mathrm{d} \sigma / \mathrm{d} Q^{2}$ together with their weighted sums for (i) suppression of the resolved cross section and for (ii) additional suppression of DIR IS, using SaS1D virtual photon PDFs [30].

i.e. being unsuppressed, in order to achieve the cancellation of the slicing parameter $\left(y_{s}\right)$ dependence of the complete NLO correction in the limit of very small $Q^{2}$ or equivalently very large $s$. It is clear that the suppression of this part of the NLO correction to the direct cross section will change the full cross section only very little as long as we choose $M_{\gamma} \simeq p_{T}^{*}$. The first term in Eq. (2), which has the suppression factor $R$, will be denoted by $\mathrm{DIR}_{\mathrm{IS}}$ in the following.

To study the left-over $M_{\gamma}$-dependence of the physical cross section, we have calculated the diffractive dijet cross section with the same kinematic constraints as in the $\mathrm{H} 1$ experiment [27]. Jets are defined by the CDF cone algorithm with jet radius equal to one and asymmetric cuts for the transverse momenta of the two jets required for infrared stable comparisons with the NLO calculations [28]. The original H1 analysis actually used a symmetric cut of $4 \mathrm{GeV}$ on the transverse momenta of both jets [29]. The data have, however, been reanalyzed for asymmetric cuts [27].

For the NLO resolved virtual photon predictions, we have used the PDFs SaS1D [30] and transformed them from the DIS ${ }_{\gamma}$ to the $\overline{\mathrm{MS}}$ scheme as in Ref. [17]. If not stated otherwise, the renormalization and factorization scales at the pomeron and the photon vertex are equal and fixed to $p_{T}^{*}=p_{T, j e t 1}^{*}$. We include four flavors, i.e. $n_{f}=4$ in the formula for $\alpha_{s}$ and in the PDFs of the pomeron and the photon. With these assumptions we have calculated the same cross section as in our previous work [15]. First we investigated how the cross section $\mathrm{d} \sigma / \mathrm{d} Q^{2}$ depends on the factorization scheme of the PDFs for the virtual photon, i.e. $\mathrm{d} \sigma / \mathrm{d} Q^{2}$ is calculated for the choice $\mathrm{SaS} 1 \mathrm{D}$ and $\mathrm{SaS} 1 \mathrm{M}$. Here $\mathrm{d} \sigma / \mathrm{d} Q^{2}$ is the full cross section (sum of direct and resolved) integrated over the momentum and rapidity ranges as in the H1 analysis. The results, shown in Fig. 2 of Ref. [26], demonstrate that the choice of the factorization scheme of the virtual photon PDFs has negligible influence on $\mathrm{d} \sigma / \mathrm{d} Q^{2}$ for all considered $Q^{2}$. The predictions agree reasonably well with the preliminary $\mathrm{H} 1$ data [27].

We now turn to the $M_{\gamma}$-dependence of the cross section with a suppression factor for DIR IS. To show this dependence for the two suppression mechanisms, (i) suppression of the resolved cross section only and (ii) additional suppression of the DIR IS term as defined in Eq. (2) in the NLO correction of the direct cross section, we consider $\mathrm{d} \sigma / \mathrm{d} Q^{2}$ for the lowest $Q^{2}$-bin, $Q^{2} \in[4,6] \mathrm{GeV}^{2}$. In Fig. 9, this cross section is plotted as a function of $\xi=M_{\gamma} / p_{T}^{*}$ in the range $\xi \in[0.25 ; 4]$ for the cases (i) (light full curve) and (ii) (full curve). We see that the cross section for case (i) has an appreciable $\xi$-dependence in the considered $\xi$ range of the order of $40 \%$, which is caused by the suppression of the resolved contribution only. With the additional suppression of the DIR IS term in the direct NLO 
correction, the $\xi$-dependence of $\mathrm{d} \sigma / \mathrm{d} Q^{2}$ is reduced to approximately less than $20 \%$, if we compare the maximal and the minimal value of $\mathrm{d} \sigma / \mathrm{d} Q^{2}$ in the considered $\xi$ range. The remaining $\xi$-dependence is caused by the NLO corrections to the suppressed resolved cross section and the evolution of the virtual photon PDFs. How the compensation of the $M_{\gamma}$-dependence between the suppressed resolved contribution and the suppressed direct NLO term works in detail is exhibited by the dotted and dasheddotted curves in Fig. 9. The suppressed resolved term increases and the suppressed direct NLO term decreases by approximately the same amount with increasing $\xi$. In addition we show also $\mathrm{d} \sigma / \mathrm{d} Q^{2}$ in the DIS theory, i.e. without subtraction of any $\ln Q^{2}$ terms (dashed line). Of course, this cross section must be independent of $\xi$. This prediction agrees very well with the experimental point, whereas the result for the subtracted and suppressed theory (full curve) lies slightly below. We notice, that for $M_{\gamma}=p_{T}^{*}$ the additional suppression of DIR IS has only a small effect. It increases $\mathrm{d} \sigma / \mathrm{d} Q^{2}$ by $5 \%$ only.

\section{Summary}

Experimental data from the $\mathrm{H} 1$ and ZEUS Collaborations at HERA for diffractive dijet production in DIS and photoproduction have been compared with NLO QCD predictions using diffractive parton densities from $\mathrm{H} 1$ and ZEUS. While good agreement was found for DIS assuming the H1 diffractive PDFs, the dijet photoproduction data are overestimated by the NLO theory, showing that factorization breaking occurs at this order. While this is expected theoretically for resolved photoproduction, the fact that the data are better described by a global suppression of direct and resolved contribution by about a factor of two has come as a surprise. We have therefore discussed in some detail the factorization scheme and scale dependence between direct and resolved contributions and proposed a new factorization scheme for diffractive dijet photoproduction.

\section{Acknowledgements}

M.K. thanks the II. Institute for Theoretical Physics at the University of Hamburg for hospitality while this work was being finalized.

\section{References}

[1] H1 Collaboration, Abstract 980, contributed to the $31^{\text {st }}$ International Conference on High Energy Physics (ICHEP 2002), Amsterdam, July 2002.

[2] S. Chekanov et al. [ZEUS Collaboration], Eur. Phys. J. C 38 (2004) 43, and A. Proskuryakov, private communication.

[3] M. Groys, A. Levy and A. Proskuryakov, these proceedings.

[4] F.-P. Schilling and P. Newman, these proceedings.

[5] A. D. Martin, M. G. Ryskin and G. Watt, Eur. Phys. J. C 37, 285 (2004) and DESY 05-055, IPPP/05/07, DCPT/05/14, hep-ph/0504132.

[6] J. C. Collins, Phys. Rev. D 57, 3051 (1998) [Erratum-ibid. D 61, 019902 (2000)].

[7] T. Affolder et al. [CDF Collaboration], Phys. Rev. Lett. 84, 5043 (2000).

[8] A. B. Kaidalov, V. A. Khoze, A. D. Martin and M. G. Ryskin, Eur. Phys. J. C 21, 521 (2001).

[9] M. Klasen, Rev. Mod. Phys. 74, 1221 (2002).

[10] A. B. Kaidalov, V. A. Khoze, A. D. Martin and M. G. Ryskin, Phys. Lett. B 567, 61 (2003).

[11] M. Klasen and G. Kramer, hep-ph/0401202, Proceedings of the $12^{\text {th }}$ International Workshop on Deep Inelastic Scattering (DIS 2004), eds. D. Bruncko, J. Ferencei and P. Strizenec, Kosice, Inst. Exp. Phys. SAS, 2004, p. 492.

[12] M. Klasen and G. Kramer, Eur. Phys. J. C 38, 93 (2004).

[13] H1 Collaboration, Abstract 6-0177, contributed to the $32^{\text {nd }}$ International Conference on High Energy Physics (ICHEP 2004), Beijing, August 2004. 
[14] ZEUS Collaboration, Abstract 6-0249, contributed to the $32^{\text {nd }}$ International Conference on High Energy Physics (ICHEP 2004), Beijing, August 2004.

[15] M. Klasen and G. Kramer, Phys. Rev. Lett. 93, 232002 (2004).

[16] D. Bödeker, G. Kramer and S. G. Salesch, Z. Phys. C 63, 471 (1994).

[17] M. Klasen, G. Kramer and B. Pötter, Eur. Phys. J. C 1, 261 (1998).

[18] S. Catani, M.H. Seymour, Nucl. Phys. B 485 (1997) 291; erratum ibid. B510 (1997) 503.

[19] H. Jung, Comp. Phys. Commun. 86 (1995) 147.

[20] ZEUS Collaboration, Abstract 295 and addendum, contributed to the $22^{\text {nd }}$ International Symposium on Lepton-Photon Interactions oh High Energy, Uppsala, Sweden, June 2005.

[21] H1 Collaboration, Abstract 6-0178, contributed to the $32^{\text {nd }}$ International Conference on High Energy Physics (ICHEP 2004), Beijing, August 2004.

[22] S. Chekanov et al. [ZEUS Collaboration], Nucl. Phys. B 672 (2003) 3,

[23] S. Frixione, Z. Kunszt and A. Signer, Nucl. Phys. B 467 (1996) 399;

S. Frixione, Nucl. Phys. B 507 (1997) 295.

[24] ZEUS Collaboration, Abstract 293, contributed to the $22^{\text {nd }}$ International Symposium on LeptonPhoton Interactions oh High Energy, Uppsala, Sweden, June 2005.

[25] B. Pötter, Comput. Phys. Commun. 133, 105 (2000).

[26] M. Klasen and G. Kramer, hep-ph/0506121, accepted for publication in J. Phys. G.

[27] S. Schätzel, hep-ex/0408049, Proceedings of the $12^{\text {th }}$ International Workshop on Deep Inelastic Scattering (DIS 2004), eds. D. Bruncko, J. Ferencei and P. Strizenec, Kosice, Inst. Exp. Phys. SAS, 2004, p. 529; H1 Collaboration, Abstract 6-0176, contributed to the $32^{\text {nd }}$ International Conference on High Energy Physics (ICHEP 2004), Beijing, August 2004.

[28] M. Klasen and G. Kramer, Phys. Lett. B 366, 385 (1996).

[29] C. Adloff et al. [H1 Collaboration], Eur. Phys. J. C 20, 29 (2001).

[30] G. A. Schuler and T. Sjöstrand, Phys. Lett. B 376, 193 (1996). 